\title{
SOLAR ROTATION AND LARGE-SCALE FLOWS MEASURED BY TIME-DISTANCE HELIOSEISMOLOGY FROM MDI
}

\author{
P. M. GILES \\ Dept of Applied Physics, Stanford University \\ Stanford, CA 94305-4085 USA \\ AND \\ T. L. DUVALL, JR. \\ Laboratory for Astronomy and Solar Physics, \\ NASA/Goddard Space Flight Center, Greenbelt, MD 20771 USA
}

\begin{abstract}
The technique of time-distance helioseismology provides a new tool for examining large-scale flows beneath the sun's surface. We have used this technique to study the meridional flow and have found that the poleward flow observed at the surface appears to persist to a depth of at least $26,000 \mathrm{~km}$. We have also examined the differential rotation in these uppermost layers, and find a slight asymmetry between the northern and southern hemispheres.
\end{abstract}

\section{Introduction}

Time-distance helioseismology relies on the measurement of wave travel times between points on the solar surface. These times can then be used to determine the properties of the solar interior. In the simplest approach, a wave is considered to travel along a narrow ray path between reflection points at the photosphere. Any material motion $\mathbf{v}$ will cause a difference in travel time, $\delta \tau$, between a wave travelling in one direction along the ray path, and one travelling in the opposite direction:

$$
\delta \tau \simeq-2 \int_{\Gamma} \frac{\mathbf{v} \cdot \hat{\mathbf{n}}}{c^{2}} d s .
$$

Here $s$ is the distance along the ray path, $\hat{\mathbf{n}}$ is the tangent to the path, and $c$ is the sound speed. The ray path $\Gamma$ is determined by the distance $\Delta$ between the end points; waves which travel a larger distance penetrate more deeply into the interior.

The travel times, therefore, can be used to measure flows beneath the solar surface. The most prominent solar flow, namely the rotation, has already been accurately measured from analysis of the normal mode frequencies (Thompson et al., 1996). However, 
the modal approach only measures the mean rotation in the northern and southern hemispheres. The time-distance method should be able to detect any asymmetry that might exist (Duvall et al., 1997a). In addition, a time-distance analysis can be used to measure the flow in meridian planes, known as the meridional circulation (Giles $e t$ al., 1997).

\section{Results}

We used observations covering one solar rotation, during the month of June, 1996. The data used were full-disk doppler images of the sun from the Michelson Doppler Imager (MDI) on SOHO. For each image, a region of the solar surface spanning 100 degrees in longitude and extending to 80 degrees latitude in each hemisphere was remapped onto a rectangular grid. The one-month time series of remapped images was divided into eight-hour segments, for the purpose of computing temporal cross correlations.

In order to study flows in the north-south direction, the cross correlations were computed for pairs of points having a common longitude. Similarly, to study flows in the east-west direction, we chose pairs of points with the same latitude. In either case, the cross correlations for all eight-hour segments during the month were averaged together, to determine the mean flow during one solar rotation.

From the cross correlations, the travel times $\tau$ were determined, as described in Duvall et al. (1997b), for each distance $\Delta$ and latitude $\lambda$. These were then used to compute the reciprocal travel time differences $\delta \tau$. An average of the flow velocity $\mathbf{v}$ in the plane of the ray path was computed using equation (1), by assuming that the flow was entirely horizontal and that it was constant along the ray path.

\subsection{MERIDIONAL CIRCULATION}

In figure 1 we show the results of the measurement of the north-south flow. The observed values of $\delta \tau$ have been averaged over the entire available distance range. The deepest rays included have photospheric reflection points separated by $73 \mathrm{Mm}$, and reach a maximum depth of $26.4 \mathrm{Mm}$.

A positive value of $\delta \tau$ indicates a northward flow, so figure 1a clearly indicates a poleward flow in both hemispheres. This is consistent with previous measurements of the meridional flow at the surface (LaBonte and Howard, 1982; Hathaway et al., 1996; Komm et al., 1993). The solid curve represents a least-squares fit to the measurements of the form

$$
\delta \tau=a_{1} \cos \lambda+a_{2} \sin 2 \lambda .
$$

For the average over all the available distances, the coefficients are found to be $a_{1}=(-0.40 \pm 0.04) \mathrm{s}$ and $a_{2}=(1.94 \pm 0.05) \mathrm{s}$. The coefficient $a_{2}$ corresponds to a peak poleward velocity of $(23.5 \pm 0.6) \mathrm{m} / \mathrm{s}$, which is somewhat larger than the value previously reported from surface measurements. This might be due to a real time variation in the flow. The term in $\cos \lambda$ is included to account for the southward velocity observed at the equator. This flow may result from an error in the orientation of the image on the MDI camera, but we do not have an independent method of determining the misalignment. We have therefore treated this cross-equator flow as an instrumental error, with the caveat that it could be a real solar feature.

We next divided the observations according to travel distance, in order to obtain information about the depth dependence of the flow. Four distance ranges were chosen, and in each case a function in the form of equation (2) was fitted to the data. 

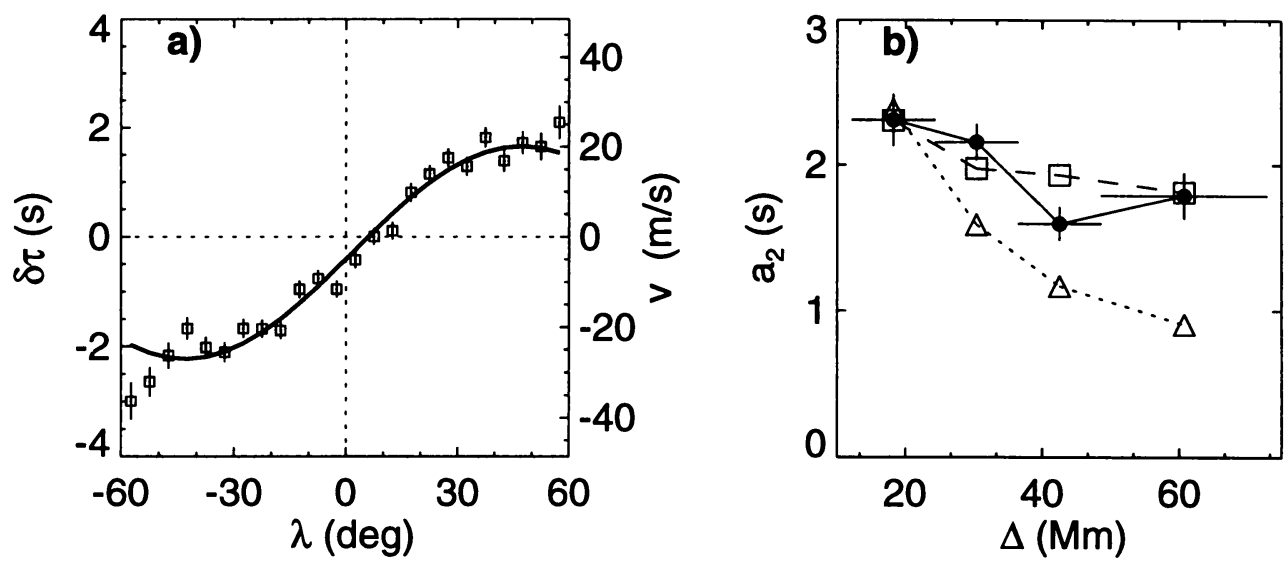

Figure 1. (a) Reciprocal travel-time differences for north-south propagating waves, as a function of latitude, and the inferred flow velocity (see text). (b) Observed travel-time differences as a function of distance travelled. The observed values are shown as the filled circles, with errors. The horizontal error bars indicate the range of distances included in the measurement for each point. Also shown are the calculated coefficients for two different flow models, both of which vary with latitude according to $v=v_{0} \sin 2 \lambda$. In one model (open squares) the velocity is constant with depth and the magnitude $v_{0}=23.5 \mathrm{~m} / \mathrm{s}$. In the second (open triangles), the flow is constant to a depth of $9.3 \mathrm{Mm}$ and zero in the deeper layers.

In figure $1 \mathrm{~b}$ the values of the coefficients $a_{2}$ are shown for the selected distances. We have compared the observational results to two simple models, described in the figure caption. For each model, the expected travel time differences were calculated using equation (1), and the function in equation (2) was fitted to the result. It seems clear that the model which confines the flow to a thin layer near the surface cannot reproduce the observations as well as the model which has a flow present throughout the region of propagation. Of course, this simple analysis does not rule out some more complicated intermediate behaviour.

\subsection{ROTATION}

Figure 2 a shows a rotation profile computed by the same method as the meridional velocity in figure 1a. For comparison, we have overplotted the surface rotation velocity (dashed curve) as obtained by Ulrich et al. (1988). Clearly, even with the simple approximations used, the measurements agree quite well. The discrepancy at the equator is consistent with helioseismic inversions which indicate that the rotation rate increases with depth very near the surface (Thompson et al., 1996).

It is also clear from figure $2 a$ that the rotation in the southern hemisphere is faster than in the northern. In figure $2 \mathrm{~b}$ we show the velocity difference at 60 degrees latitude, as a function of the travel distance. This asymmetry also appears to persist to the maximum depth of the ray penetration.

\section{Conclusions}

We believe that this is the first measurement of the global meridional flow beneath the solar surface, although previous measurements have been made over a smaller area 

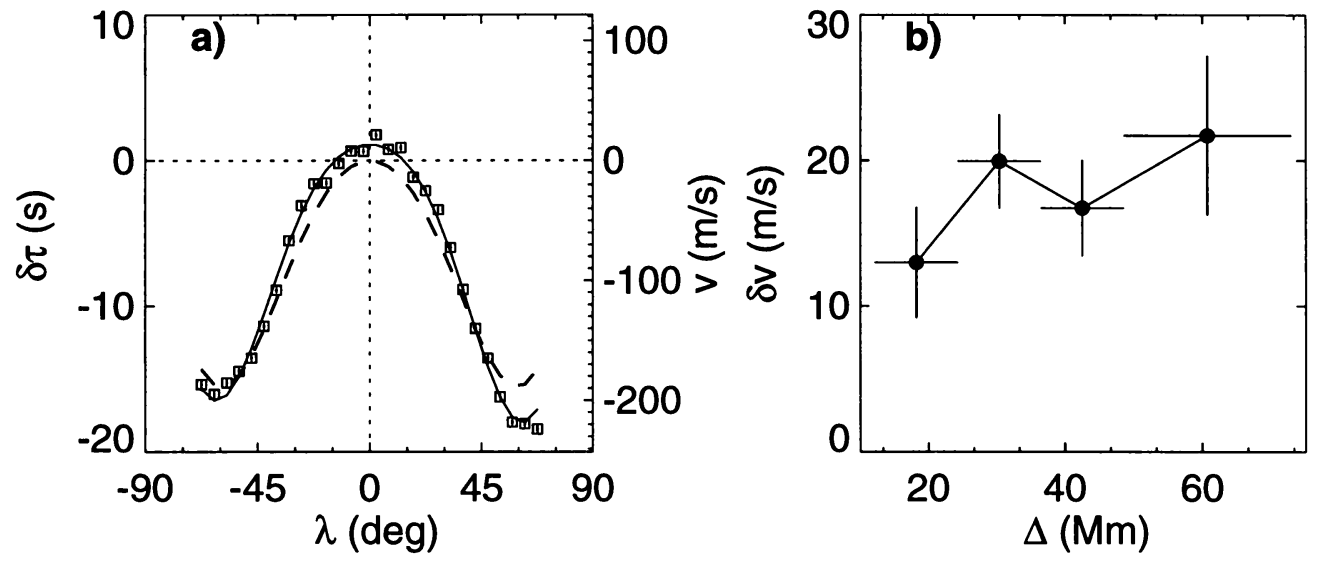

Figure 2. (a) Reciprocal travel-time differences for east-west propagating waves, as a function of latitude, and the inferred flow velocity (see text). The solid curve is a fit to the observations, and the dashed curve is the measured surface rotation rate. Note that the solid-body component of the equatorial surface rate has been subtracted from all the velocities. (b) Inferred velocity difference between northern and southern hemispheres, as a function of wave travel distance. The velocities were taken at $\pm 60^{\circ}$ latitude. The sense of the asymmetry is that the southern hemisphere is rotating more rapidly.

using a different technique (Patrón et al., 1995). Our results indicate that the poleward flow previously observed is not confined to a thin layer near the surface, but persists to a depth of at least $26 \mathrm{Mm}$. We estimate the velocity of this flow to have a maximum value of $(23.5 \pm 0.6) \mathrm{m} / \mathrm{s}$. We have also shown that the time-distance technique can be used to measure the differential rotation, and we find that the southern hemisphere is rotating slightly faster than the northern, by $10-20 \mathrm{~m} / \mathrm{s}$ at 60 degrees latitude.

The preliminary results presented here will be extended in the future to cover a longer time period, and a larger part of the solar surface. Particularly interesting will be to extend the measurements to greater depths, and to examine the time variation of the flows during the solar cycle.

We would like to acknowledge support for this research by NASA contract NAG53077 at Stanford University.

\section{References}

Duvall, T. L. Jr., Kosovichev, A. G., and Scherrer, P. H. (1997a) in Sounding Solar and Stellar Interiors, in press.

Duvall, T. L. Jr.,et al. (1997b) Solar Physics 170, pp. 63-73.

Giles, P. M., Duvall, T. L. Jr., Scherrer, P. H., and Bogart, R. S. (1997) Nature, in press.

Hathaway, D. H. et al. (1996) Science 272, pp. 1306-1309.

Komm, R. W., Howard, R. F., and Harvey, J. W. (1993) Solar Physics 147, pp. 207-223.

LaBonte, B. J. and Howard, R. F. (1982) Solar Physics 80, pp. 361-372.

Patrón, J., Hill, F., Rhodes, E. J. Jr., Korzennik, S. G., and Cacciani, A. (1995) Ap. J. 455, pp. 746-757.

Thompson, M. J. et al. (1996) Science 272, pp. 1300-1305.

Ulrich, R. K., Boyden, J. E., Webster, L., Padilla, S. P., Gilman, P., and Sheiber, T. (1988) Solar Physics 117, p. 291. 